\title{
Gravitational waves from Pati-Salam dynamics
}

\author{
W. C. Huang, ${ }^{1}$ F. Sannino, ${ }^{1,2,3}$ and Z. W. Wang $\odot^{1, *}$ \\ ${ }^{1}$ CP $P^{3}$-Origins, University of Southern Denmark, Campusvej 555230 Odense M, Denmark \\ ${ }^{2}$ Dipartimento di Fisica Ettore Pancini, Universit di Napoli Federico II-INFN sezione di Napoli \\ Complesso Universitario di Monte Sant'Angelo Edificio 6, via Cintia, 80126 Napoli, Italy \\ ${ }^{3}$ The Danish Institute for Advanced Study, University of Southern Denmark, \\ Campusvej 55, DK-5230 Odense M, Denmark
}

(Received 16 May 2020; accepted 3 November 2020; published 24 November 2020)

\begin{abstract}
We show that it is possible to use gravitational wave detectors to observe the occurrence of a first order phase transition in Pati-Salam extensions of the Standard Model. We show that the peak frequency of the expected gravitational wave signals ranges within $0.1-10 \mathrm{~Hz}$. We find amusing that the next generation of gravity waves detectors are able to explore time-honored extensions of the Standard Model occurring at energy scales inaccessible by present and future particle physics accelerators.
\end{abstract}

DOI: 10.1103/PhysRevD.102.095025

\section{INTRODUCTION}

The idea of using gravitational wave as a complementary approach to explore particle physics started some time ago [1-3]. However, the bulk of the research concentrated, so far, on the electroweak phase transition which is typically in the detection region of the LISA gravitational wave detector as nicely summarized in $[4,5]$. Of special interest is the possible detection of gravity waves originated in grand unified theories (GUTs). The interest arises also because typically the new physics energy scale of GUTs is beyond the reach of the existing and even future $100 \mathrm{TeV}$ colliders.

A prerequisite to start even discussing gravitational wave detection is that the underlying theory must undergo a strong first order phase transition at some point during the evolution of the Universe. Additionally, the higher the energy scale of the first order phase transition, the higher the peak frequency of the gravitational wave that needs to be detected will be. Inevitably, the upper frequency limit of the existing and planned gravitational wave detectors (roughly at order of $10^{3} \mathrm{~Hz}$ ) provides an upper bound on the detectable energy scale (roughly at $10^{4}-10^{5} \mathrm{TeV}$ ). In this sense, among the different types of GUTs, only the two semisimple GUTs, the Pati-Salam (PS) model [6] and Trinification model [7], satisfy this criterion. In this work, we will focus mainly on the gravitational wave signatures of the minimal Pati-Salam model. Our investigation differs from the one in [8] in which an alternative model of the

\footnotetext{
*wang@cp3.sdu.dk
}

Published by the American Physical Society under the terms of the Creative Commons Attribution 4.0 International license. Further distribution of this work must maintain attribution to the author(s) and the published article's title, journal citation, and DOI. Funded by SCOAP ${ }^{3}$.
Pati-Salam model was considered. In that work the authors employed a rather involved matter content that featured, however, a simpler first order phase transition structure. ${ }^{1}$ Gravitational wave signatures in the $P S^{3}$ model (a generalization of the Pati-Salam model involving three copies to address the flavor hierarchies) can be found in [9].

The Pati-Salam model of matter field unification [6] is a time-honored example in which one can address the hypercharge triviality issue by embedding it in an asymptotically free theory. From a phenomenological standpoint it can be commended because it does not induce fast proton decay, and it can even be extended to provide a stable proton [10] while automatically providing a rationale for the existence of right-handed neutrinos (see more details in a recent nice review [11]) In addition, it can be shown that primordial monopoles induced by the phase transition of Pati-Salam symmetry breaking at such low scales (of order $1000 \mathrm{TeV}$ ) are significantly suppressed [12-14]. Thus, our work is not plagued with the monopole problem. ${ }^{2}$

\footnotetext{
${ }^{1}$ In the work of [8], the authors try to realize the gauge coupling unification and symmetry breaking to an intermediate step (left-right model) first and thus their scalar sectors are overall more complicated. However, their first order phase transition occurs only when $S U(4)$ is breaking, while in our case both $S U(4)$ and $S U(2)_{R}$ break and thus their analysis of the first order phase transition is simpler and fewer couplings are involved.

${ }^{2}$ The monopoles are created during the phase transition from symmetry breaking $G_{\mathrm{PS}} \rightarrow G_{\mathrm{SM}}$ in light of the Abelian $\mathrm{SM} U(1)_{Y}$, which does not individually appear (but is embedded instead) in $G_{\mathrm{PS}}, S U(4) \otimes S U(2)_{L} \otimes S U(2)_{R}$. However, it can be shown that [12-14] the number density to entropy ratio of the monopole is $n_{M} / s \sim\left[\left(\frac{T_{c}}{M_{\mathrm{pl}}}\right) \ln \left(\frac{m_{\mathrm{pl}}^{4}}{T_{c}^{4}}\right)\right]^{3}$ where $T_{c}$ is the critical temperature during the phase transition and $m_{\mathrm{pl}}$ is the Planck mass. That results in a negligible monopole density, given $T_{c} \sim 10^{6} \mathrm{GeV}$.
} 
So far, asymptotic freedom has been the well-traveled route to resolve the triviality problem. An alternative route is that in which the UV theory acquires an interacting fixed point, before gravity sets in, de facto saving itself from the presence of a cutoff. This unexplored route was opened when the first safe gauge-Yukawa theory was discovered in [15].

To achieve a safe theory with a small number of colors we employ a large number of matter fields techniques $[16,17]$. The first phenomenological applications of the large- $N_{f}$ limit appeared in [18] where it was first explored whether the Standard Model (SM) augmented by a large number of vectorlike fermions can have an ultraviolet fixed point in all couplings. The full treatment appeared in [19] and was further generalized in [20]. It was found in [19] and later on proved in [20] that while the non-Abelian gauge couplings, Higgs quartic and Yukawa coupling, can exhibit a safe fixed point, the hypercharge remains troublesome. In fact, for Abelian theories, the fermion mass anomalous dimension diverges at the alleged fixed point [21] suggesting that a safe extension of the SM, like the asymptotically free counterpart, is best obtained by embedding the SM in a non-Abelian gauge structure. The first nonAbelian safe PS and Trinification embeddings were put forward in $[22,23]$. However, in the minimal models, only one generation of SM fermions can be modeled, since all the Yukawa couplings are determined by the same UV fixed point value with no resulting hierarchy at low energy. Yukawa hierarchies among three generations of SM fermions are discussed in [24].

In this work, we will start by investigating gravitational wave signatures emerging in Pati-Salam extensions of the SM embedded in an asymptotically safe scenario. We use these predictions as an initial seed value to study the first order phase transition and gravitational wave signatures. Later, we will depart from the safety scenario and will explore a more general parameter space. Therefore, our work of studying the phase transition and gravitational wave generation is very general and applies to both safe and nonsafe embeddings of the Pati-Salam model.

We discover that the next-generation gravity wave detectors are able to explore time-honored extensions of the Standard Model occurring at energy scales inaccessible by present and future particle physics colliders. More precisely, we show that the peak frequency of the expected gravitational wave signals ranges within $0.1-10 \mathrm{~Hz}$.

The paper is organized as follows. In Sec. II we introduce the Pati-Salam model while in Sec. III we compute the finite-temperature corrections to the relevant part of the potential of the theory. The order of the phase transition as well as gravitational wave generation and detection are studied in Sec. IV. The predictions for the gravity wave signals stemming from the model parameters are presented in Sec. V. We conclude in Sec. VI. In the Appendix we provide some detailed computations.

\section{INTRODUCING THE PATI-SALAM MODEL}

We first briefly review the Pati-Salam embedding of the SM suggested in [22].

Consider the time-honored PS gauge symmetry group $G_{\mathrm{PS}}[6]$

$$
G_{\mathrm{PS}}=S U(4) \otimes S U(2)_{L} \otimes S U(2)_{R},
$$

with gauge couplings $g_{4}, g_{L}$, and $g_{R}$, respectively. Here the gauge group $S U(4) \supset S U(3)_{C} \otimes U(1)_{B-L}$, where $S U(3)_{C}$ denotes the SM QCD gauge group. The SM quark and lepton fields are unified into the $G_{\mathrm{PS}}$ irreducible representations

$$
\begin{aligned}
\psi_{L i} & =\left(\begin{array}{llll}
u_{L}^{1} & u_{L}^{2} & u_{L}^{3} & \nu_{L} \\
d_{L}^{1} & d_{L}^{2} & d_{L}^{3} & e_{L}
\end{array}\right)_{i} \sim(4,2,1)_{i}, \\
\psi_{R i} & =\left(\begin{array}{llll}
u_{R}^{1} & u_{R}^{2} & u_{R}^{3} & \nu_{R} \\
d_{R}^{1} & d_{R}^{2} & d_{R}^{3} & e_{R}
\end{array}\right)_{i} \sim(4,1,2)_{i},
\end{aligned}
$$

where $i=1,2,3$ is a flavor index. In order to induce the breaking of $G_{\mathrm{PS}}$ to the SM gauge group, we introduce a scalar field $\phi_{R}$ which transforms as the fermion multiplet $\psi_{R}$, that is, $\phi_{R} \sim(4,1,2)$ :

$$
\phi_{R}=\left(\begin{array}{cccc}
\phi_{R}^{u_{1}} & \phi_{R}^{u_{2}} & \phi_{R}^{u_{3}} & \phi_{R}^{0} \\
\phi_{R}^{d_{1}} & \phi_{R}^{d_{2}} & \phi_{R}^{d_{3}} & \phi_{R}^{-}
\end{array}\right),
$$

where the neutral component $\phi_{R}^{0}$ takes a nonzero vacuum expectation value, $\left\langle\phi_{R}^{0}\right\rangle \equiv v_{R}$, such that $G_{\mathrm{PS}} \stackrel{v_{R}}{\rightarrow} S U(3)_{C} \otimes$ $S U(2)_{L} \otimes U(1)_{Y}$. We also introduce an additional (complex) scalar field $\Phi \sim(1,2,2)$, with

$$
\Phi=\left(\begin{array}{cc}
\phi_{1}^{0} & \phi_{2}^{+} \\
\phi_{1}^{-} & \phi_{2}^{0}
\end{array}\right) \equiv\left(\begin{array}{ll}
\Phi_{1} & \Phi_{2}
\end{array}\right),
$$

which is responsible of the breaking of the electroweak symmetry. After Pati-Salam symmetry breaking, the bidoublet scalar field becomes the two Higgs doublet fields. The spontaneous symmetry breaking of the two Higgs doublet scalar sector breaks the electroweak symmetry of the Standard Model.

The most general Yukawa Lagrangian for the matter fields $\psi_{L / R}$ is

$$
\mathcal{L}_{\text {Yuk }}^{\psi}=y \operatorname{Tr}\left[\overline{\psi_{L}} \Phi \psi_{R}\right]+y_{c} \operatorname{Tr}\left[\overline{\psi_{L}} \Phi^{c} \psi_{R}\right]+\text { H.c. },
$$

where $y$ and $y_{c}$ are the Yukawa couplings for the third generation only. Note that the Yukawa couplings of the first two generations can be generated through the clockwork mechanism [24].

In this work, we consider the case of a self-conjugate bidoublet field $\Phi \equiv \Phi^{c}$ which known as the minimal PatiSalam model put forward first in [14]. The beauty of this minimal model is the occurrence of a further matter unification according to which one obtains degenerate 
TABLE I. Gauge, Yukawa, and scalar quartic couplings of the PS model.

\begin{tabular}{lcc}
\hline \hline Gauge & Yukawa & Scalar \\
\hline$S U(4): g_{4}$ & $\psi_{L / R}: y, y_{c}$ & $\phi_{R}: \lambda_{R 1}, \lambda_{R 2}$ \\
$S U(2)_{L}: g_{L}$ & $N_{L}: y_{\nu}$ & portal: $\lambda_{R \Phi_{1}}, \lambda_{R \Phi_{2}}, \lambda_{R \Phi_{3}}$ \\
$S U(2)_{R}: g_{R}$ & $F: y_{F}$ & $\Phi: \lambda_{1}, \lambda_{2}, \lambda_{3}, \lambda_{4}$ \\
\hline \hline
\end{tabular}

masses, at tree level, above the Pati-Salam symmetry breaking scale, namely,

$$
m_{t}=m_{b}=m_{\tau}=m_{\nu_{\tau}}
$$

where $m_{t}, m_{b}, m_{\tau}, m_{\nu_{\tau}}$ denote the masses of the top, bottom, tau lepton, and right-handed neutrino. The price to pay is the need to introduce a chiral fermion singlet $N_{L}(1,1,1)$ and extra vectorlike fermions $F \sim(10,1,1)$ in order to trigger the mass splitting below the Pati-Salam symmetry breaking scale (see below for more detailed discussions). In order to separate the neutrino and top masses in Eq. (6), we implement the inverse seesaw mechanism ${ }^{3}[25-28]$ by adding a new chiral fermion singlet $N_{L} \sim(1,1,1),{ }^{4}$ which has the Yukawa interaction (see, e.g., $[14,22]$ for more details)

$$
\mathcal{L}_{\text {Yuk }}^{N}=-y_{\nu} \overline{N_{L}} \operatorname{Tr}\left[\phi_{R}^{\dagger} \psi_{R}\right]+\text { H.c. }
$$

In order to split the mass of the top, bottom, and tau lepton in Eq. (6), we introduce a new vectorlike fermion multiplet $F \sim(10,1,1)$ with mass $M_{F}$ and Yukawa interactions (see, e.g., $[14,22]$ for more details):

$$
\mathcal{L}_{\text {Yuk }}^{F}=y_{F} \operatorname{Tr}\left(\overline{F_{L}} \phi_{R}^{T} i \tau_{2} \psi_{R}\right)+\text { H.c. }
$$

All the field contents and couplings are summarized in Table I.

\section{FINITE TEMPERATURE EFFECTIVE POTENTIAL}

\section{A. Tree-level effective potential of the Pati-Salam model}

The general scalar potential of the model defined above is given by

\footnotetext{
${ }^{3}$ The inverse seesaw mechanism has the other advantage to introduce big hierarchies between left and right neutrino masses while no ultra high symmetry breaking scale is required. Note that a traditional seesaw mechanism without an extra gauge singlet $N_{L}(1,1,1)$ implies a much larger symmetry breaking scale $10^{13} \mathrm{GeV}[14,25]$.

${ }^{4}$ In this work, we focus on the third generation, since the first two generations with small couplings give rise to negligible thermal corrections and hence are ignored. Thus, we consider a single generation for each of the three types of Weyl neutrinos $\nu_{L}$, $\nu_{R}, N_{L}$.
}

$$
\begin{aligned}
V\left(\Phi, \phi_{R}\right)= & \lambda_{1} \operatorname{Tr}^{2}\left(\Phi^{\dagger} \Phi\right)+\operatorname{Re}\left[\lambda_{2} \operatorname{Tr}^{2}\left(\Phi^{\dagger} \Phi^{c}\right)\right] \\
& +\operatorname{Re}\left[\lambda_{3} \operatorname{Tr}\left(\Phi^{\dagger} \Phi\right) \operatorname{Tr}\left(\Phi^{\dagger} \Phi^{c}\right)\right] \\
& +\left(\lambda_{4}-2 \operatorname{Re} \lambda_{2}\right)\left|\operatorname{Tr}\left(\Phi^{\dagger} \Phi^{c}\right)\right|^{2} \\
& +\lambda_{R 1} \operatorname{Tr}^{2}\left(\phi_{R}^{\dagger} \phi_{R}\right)+\lambda_{R 2} \operatorname{Tr}\left(\phi_{R}^{\dagger} \phi_{R} \phi_{R}^{\dagger} \phi_{R}\right) \\
& +\lambda_{R \Phi 1} \operatorname{Tr}\left(\phi_{R}^{\dagger} \phi_{R}\right) \operatorname{Tr}\left(\Phi^{\dagger} \Phi\right) \\
& +\lambda_{R \Phi 3} \operatorname{Tr}\left(\phi_{R}^{\dagger} \phi_{R} \Phi^{\dagger} \Phi\right) \\
& +\operatorname{Re}\left[\lambda_{R \Phi_{2}} \operatorname{Tr}\left(\phi_{R} \phi_{R}^{\dagger}\right) \operatorname{Tr}\left(\Phi^{\dagger} \Phi^{c}\right)\right] .
\end{aligned}
$$

Since in this work we focus on the study of the phase transition at the Pati-Salam symmetry breaking scale, the terms involving $\Phi$ can be safely ignored and we focus on the relevant terms involving $\phi_{R}$ only in Eq. (9) above. Below, we write down only the relevant potential terms:

$V_{\text {tree }}\left(\phi_{R}\right)=\lambda_{R 1} \operatorname{Tr}^{2}\left(\phi_{R}^{\dagger} \phi_{R}\right)+\lambda_{R 2} \operatorname{Tr}\left(\phi_{R}^{\dagger} \phi_{R} \phi_{R}^{\dagger} \phi_{R}\right)$,

while the canonically normalized kinetic term with the gauge covariant derivative reads

$$
K=\operatorname{Tr}\left(D_{\mu} \phi_{R}^{\dagger} D^{\mu} \phi_{R}\right),
$$

with

$D^{\mu} \phi_{R}=\partial^{\mu} \phi_{R}+i g_{R}\left(W_{R}^{\mu} \cdot T_{R}\right) \phi_{R}-i g_{4} \phi_{R}\left(A^{\mu} \cdot T_{4}\right)$,

where $g_{4}\left(g_{R}\right)$ represents the $S U(4)\left(S U(2)_{R}\right)$ gauge coupling. The inner products of the gauge bosons and corresponding generators for $S U(4)$ and $S U(2)_{R}$ are denoted by $\left(A \cdot T_{4}\right)$ and $\left(W_{R} \cdot T_{R}\right)$ with $\operatorname{Tr}\left(T_{R}^{i} T_{R}^{j}\right)=\operatorname{Tr}\left(T_{4}^{i} T_{4}^{j}\right)=$ $\delta^{i j} / 2$. It is important to note that we do not include any explicit mass terms in the tree-level potential. The symmetry breaking in this work is induced by the ColemanWeinberg mechanism. We are interested in the model with classical scale invariance for two reasons. First, without the mass parameter, the predictive power of the model is increased. The Pati-Salam symmetry breaking scale in this work is an outcome rather than being inserted by hand. Starting from UV (either with or without fixed point), the renormalization group (RG) flow runs from UV to IR until reaching a certain scale where dynamical Pati-Salam symmetry breaking happens, further triggering the gravitational wave. Second, we would like to further explore the compatibility between asymptotic safety and ColemanWeinberg symmetry breaking in this model. ${ }^{5}$

If we write out $\phi_{R}$ explicitly as

$$
\frac{1}{\sqrt{2}}\left(\begin{array}{cccc}
\phi_{R 1}+i \phi_{R 2} & \cdots & \cdots & \phi_{R 7}+i \phi_{R 8} \\
\phi_{R 9}+i \phi_{R 10} & \cdots & \cdots & v+\phi_{R 15}+i \phi_{R 16}
\end{array}\right),
$$

\footnotetext{
${ }^{5}$ Preliminary studies investigating the compatibility between the occurrence of asymptotic freedom and Coleman-Weinberg symmetry breaking was shown in $[29,30]$ and investigated more recently in [31].
} 
where we choose the symmetry breaking direction of $\phi_{R}$ and, thus, all field components except the $\phi_{R 15}$ direction are zero. As mentioned above, $\left\langle\phi_{R}\right\rangle$ triggers the breaking of $G_{\mathrm{PS}} \stackrel{v_{R}}{\rightarrow} S U(3)_{C} \otimes S U(2)_{L} \otimes U(1)_{Y}$. Out of sixteen scalar fields, there are nine Goldstone bosons and seven physical bosons. Therefore, eight gauge bosons of $S U(4)$ (corresponding to QCD gluons) and one gauge field from $S U(4) \otimes S U(2)_{R}$ [which is simply $U(1)_{Y}$, a linear combination of the $U(1)_{B-L}$ from $S U(4)$ and $U(1)_{R}$ from $S U(2)_{R}$, with $\left.Y=2 I_{R}+B-L\right]$ remain massless. The other nine gauge bosons of $S U(4) \otimes S U(2)_{R}$ (six leptoquark, two right boson $W_{R}^{ \pm}$and one $Z^{\prime}$ ) become massive.

With Eq. (10), we can construct the mass matrix of the scalar fields and obtain sixteen tree-level mass eigenvalues. These mass eigenvalues can be divided into nine Goldstone bosons with a quadratic contribution $M_{\mathrm{Gold}}^{2}=v^{2}\left(\lambda_{\mathrm{R} 1}+\lambda_{\mathrm{R} 2}\right)^{6}$ and seven physical Higgses, one out of which has a mass of $M_{\mathrm{Higgs} 1}^{2}=3 v^{2}\left(\lambda_{\mathrm{R} 1}+\lambda_{\mathrm{R} 2}\right)$ and six other Higgses with a mass $M_{\mathrm{Higgs} 2}^{2}=v^{2} \lambda_{\mathrm{R} 1}$.

\section{B. Loop level effective potential of the Pati-Salam model}

Contributions from one-loop vacuum bubble diagrams consist of both zero- and finite-temperature parts (see, e.g., [32]). In this section, we first discuss the zero-temperature contributions to the effective potential from scalar, gauge fields, and fermions. The general formula is well known and can be written as

$$
V_{\text {lloop }}=\sum_{i} \pm n_{i} \frac{m_{i}^{4}}{64 \pi^{2}}\left(\log \left[\frac{m_{i}^{2}}{\mu^{2}}\right]-C_{i}\right)
$$

where the sum runs over the bosons (+) and fermions (-) and $n_{i}$ counts the internal degrees of freedom (d.o.f.) of each species $i$. The symbols $m_{i}, \mu$, and $C_{i}$ correspond, respectively, to the tree-level mass terms, renormalization scale, and constant (equal to 5/6 for gauge bosons and $3 / 2$ for scalars and fermions in the minimal subtraction scheme). We define the background field as $\rho$. In the following, we write out the scalars, gauge fields, and fermions contribution explicitly.

The Higgs fields contributions (7 d.o.f.) to the one-loop effective potential $V_{\text {Higgs }}$ are

\footnotetext{
${ }^{6}$ Note that in the theory with classical scale invariance, the physical goldstone masses are massless only after the symmetry is broken by the radiative loop corrections which are different from the cases without classical scale invariance that the Goldstone bosons are massless already at the tree level. What we call Goldstone bosons masses are actually not their physical masses but instead the coefficients of the quadratic contributions of the Goldstone bosons at the tree level (not at the one-loop level), which are the ones that should be used to consistently obtain the one-loop effective potential and are not zero.
}

$$
\begin{aligned}
& \frac{1}{64 \pi^{2}}\left(3 \rho^{2}\left(\lambda_{R 1}+\lambda_{R 2}\right)\right)^{2}\left(\log \left(\frac{3 \rho^{2}\left(\lambda_{R 1}+\lambda_{R 2}\right)}{\mu^{2}}\right)-\frac{3}{2}\right) \\
& +\frac{6}{64 \pi^{2}}\left(\rho^{2} \lambda_{R 1}\right)^{2}\left(\log \left(\frac{\rho^{2} \lambda_{R 1}}{\mu^{2}}\right)-\frac{3}{2}\right) .
\end{aligned}
$$

The Goldstone contributions (9 d.o.f.) to the one-loop effective potential $V_{\text {Gold }}$ are

$\frac{9}{64 \pi^{2}}\left(\rho^{2}\left(\lambda_{R 1}+\lambda_{R 2}\right)\right)^{2}\left(\log \left(\frac{\rho^{2}\left(\lambda_{\mathrm{R} 1}+\lambda_{\mathrm{R} 2}\right)}{\mu^{2}}\right)-\frac{3}{2}\right)$.

The leptoquark contributions from $S U(4)$ gauge fields $(6$ leptoquark $\times 3$ polarization $=18$ d.o.f. $)$ to the one-loop effective potential are

$$
V_{\text {lepto }}=\frac{18}{64 \pi^{2}}\left(\frac{1}{4} g_{4}^{2} \rho^{2}\right)^{2}\left(\log \left(\frac{g_{4}^{2} \rho^{2}}{4 \mu^{2}}\right)-\frac{5}{6}\right),
$$

where the tree-level leptoquark mass is given by $M_{\text {lepto }}^{2}=\frac{1}{4} g_{4}^{2} v^{2}$. The gauge boson $W_{R}^{ \pm}$contributions $\left(2 W_{R} \times 3\right.$ polarization $=6$ d.o.f. $)$ to the one-loop effective potential are

$$
V_{W_{R}^{ \pm}}=\frac{6}{64 \pi^{2}}\left(\frac{1}{4} g_{R}^{2} \rho^{2}\right)^{2}\left(\log \left(\frac{g_{R}^{2} \rho^{2}}{4 \mu^{2}}\right)-\frac{5}{6}\right),
$$

where the tree-level $W_{R}$ mass is given by $M_{W_{R}^{ \pm}}^{2}=\frac{1}{4} g_{R}^{2} v^{2}$.

The $Z^{\prime}$ boson contribution $\left(1 Z^{\prime} \times 3\right.$ polarization $=$ 3 d.o.f.) to the one-loop effective potential $V_{Z^{\prime}}$ is

$$
\frac{3}{64 \pi^{2}}\left(\frac{1}{8}\left(2 g_{R}^{2}+3 g_{4}^{2}\right) \rho^{2}\right)^{2}\left(\log \left(\frac{\left(2 g_{R}^{2}+3 g_{4}^{2}\right) \rho^{2}}{8 \mu^{2}}\right)-\frac{5}{6}\right),
$$

where the tree-level $Z^{\prime}$ mass is given by $M_{Z^{\prime}}^{2}=$ $\frac{1}{8}\left(2 g_{R}^{2}+3 g_{4}^{2}\right) v^{2}$.

The neutrino singlet contribution (4 d.o.f. of Dirac Fermion) to the one-loop effective potential is

$$
V_{\nu}=-\frac{4}{64 \pi^{2}}\left(\frac{1}{2} y_{\nu}^{2} \rho^{2}\right)^{2}\left(\log \left(\frac{y_{\nu}^{2} \rho^{2}}{2 \mu^{2}}\right)-\frac{3}{2}\right),
$$

where the tree-level neutrino singlet mass is given by $M_{\nu}^{2}=\frac{1}{2} y_{\nu}^{2} v^{2}$.

On the other hand, the Yukawa coupling in Eq. (8) also contributes to the potential as $(4$ colors $\times 4$ d.o.f. of Dirac fermion $=16$ d.o.f.)

$$
V_{\mathrm{F}}=-\frac{16}{64 \pi^{2}}\left(\frac{1}{2} y_{F}^{2} \rho^{2}\right)^{2}\left(\log \left(\frac{y_{F}^{2} \rho^{2}}{2 \mu^{2}}\right)-\frac{3}{2}\right),
$$

with a mass term $M_{F}^{2}=\frac{1}{2} y_{F}^{2} v^{2}$. All in all, the total one-loop effective potential is

$$
V_{\text {lloop }}=V_{\text {Higgs }}+V_{\text {Gold }}+V_{\text {lepto }}+V_{W_{R}^{ \pm}}+V_{Z^{\prime}}+V_{\nu}+V_{F} \text {. }
$$




\section{Finite-temperature effective potential of the Pati-Salam model}

The one-loop finite-temperature effective potential has the following general form (see, e.g., [33]):

$V_{T}=\sum_{i} \pm n_{i} \frac{T^{4}}{2 \pi^{2}} \int_{0}^{\infty} d y y^{2} \log \left[1 \mp e^{-\sqrt{y^{2}+m_{i}^{2} / T^{2}}}\right]$,

where $+n_{i}\left(-n_{i}\right)$ corresponds to bosons (fermions). We can further write the thermal integral in the form of the polynomials which can significantly simplify the calculations. We focus on the integral part of Eq. (23) and define

$$
I_{B, F}(a)= \pm \int_{o}^{\infty} d y y^{2} \log \left[1 \mp e^{-\sqrt{y^{2}+a}}\right],
$$

where we have used $a \equiv m_{i}^{2} / T^{2}$. For high temperature expansions $\left(m_{i} / T \ll 1\right)$, the thermal integral can be expanded respectively for bosons and fermions as

$I_{B}^{H}(a)=-\frac{\pi^{4}}{45}+\frac{\pi^{2}}{12} a-\frac{\pi}{6} a^{\frac{3}{2}}-\frac{a^{2}}{32}\left(\log (a)-c_{B}\right)$

$I_{F}^{H}(a)=-\frac{7 \pi^{4}}{360}+\frac{\pi^{2}}{24} a+\frac{a^{2}}{32}\left(\log (a)-c_{F}\right)$,

where $c_{B}$ and $c_{F}$ are, respectively, $c_{B}=3 / 2-2 \gamma_{E}+$ $2 \log (4 \pi) \quad$ and $c_{F}=3 / 2-2 \gamma_{E}+2 \log (\pi)$ and $\gamma_{E} \approx$ 0.5772. For low temperature expansions $\left(m_{i} / T \gg 1\right)$, the thermal integral for both bosons and fermions can be expanded as ${ }^{7}$

$$
I_{B, F}^{L}(a)=-\sqrt{\frac{\pi}{2}} a^{\frac{3}{4}} e^{-\sqrt{a}}\left(1+\frac{15}{8} a^{-\frac{1}{2}}+\frac{105}{128} a^{-1}\right) .
$$

To include the information for both the high temperature and low temperature, we need to have an expression to connect the above two expressions, Eq. (25) and Eq. (26). We find

$$
\begin{aligned}
& I_{B}(a)=e^{-\left(\frac{a}{6.3}\right)^{4}} I_{B}^{H}(a)+\left(1-e^{-\left(\frac{a}{6.3}\right)^{4}}\right) I_{B}^{L} \\
& I_{F}(a)=e^{-\left(\frac{a}{3.25}\right)^{4}} I_{F}^{H}(a)+\left(1-e^{-\left(\frac{a}{3.25}\right)^{4}}\right) I_{F}^{L} .
\end{aligned}
$$

Thus, we have the finite-temperature effective potential (without ring contributions so far) as

$$
\begin{aligned}
V_{T}^{\mathrm{tot}}= & \frac{T^{4}}{2 \pi^{2}}\left(I_{B}\left[\frac{M_{\mathrm{Higgs} 1}^{2}}{T^{2}}\right]+6 I_{B}\left[\frac{M_{\text {Higgs } 2}^{2}}{T^{2}}\right]\right. \\
& +9 I_{B}\left[\frac{M_{\text {Gold }}^{2}}{T^{2}}\right]+6 I_{B}\left[\frac{M_{W_{R}^{ \pm}}^{2}}{T^{2}}\right]+3 I_{B}\left[\frac{M_{Z^{\prime}}^{2}}{T^{2}}\right] \\
& \left.+18 I_{B}\left[\frac{M_{\text {lepto }}^{2}}{T^{2}}\right]+4 I_{F}\left[\frac{M_{\nu}^{2}}{T^{2}}\right]+16 I_{F}\left[\frac{M_{F}^{2}}{T^{2}}\right]\right) .
\end{aligned}
$$

\footnotetext{
${ }^{7}$ Note that there are typos in the expressions of the low energy expansion in [34].
}

\section{Ring contribution to the effective potential of the Pati-Salam model}

The next-leading contributions go beyond the mean-field approximation by including the finite-temperature corrections (characterized by the two-point function) to the propagator in the one-loop vacuum bubble diagram; see, e.g., [32]. The two-point function receives contributions from both scalars and vector bosons. The idea is to sum over all relevant diagrams (so-called Daisy or ring diagrams) with insertions of one, two, $\ldots, N(N \rightarrow \infty)$ twopoint functions, respectively, on top of the leading-order propagator. Pictorially, the insertions can be represented by small rings attached to the main ring (propagator).

The general formula for the ring contributions can be written as

$V_{\text {ring }}^{i}=-\frac{T}{12 \pi}\left(\left[m_{i}^{2}(\rho)+\sum_{\text {bosons } j} \pi_{i}^{j}(0)\right]^{3 / 2}-m_{i}^{3}(\rho)\right)$,

where $\pi_{i}(0)$ denotes the corresponding thermal mass contributions to the species $i$ from the relevant bosonic d.o.f. $j$ (in the outside rings of the daisy diagram). To consider the ring diagram contributions to the Higgs field, for example, $\pi_{\text {Higgs }}$ should include all the scalar field (thermal mass) contributions denoted as $\pi_{\text {Higgs }}^{\text {Higgs }}, \pi_{\text {Higgs }}^{\text {Higgs2 }}$, $\pi_{\mathrm{Higgs}}^{\mathrm{Gold}}$ as well as the gauge field contributions. For thermal mass contributions to the scalar field from the gauge and scalar fields (i.e., scalar field in the big central ring of the Daisy diagram), we have the following general formula for the contributions of different species $j$ in the outside ring of the daisy diagram, i.e.,

$$
\pi_{\text {scalar }}^{j}(0)=\frac{1}{12} \frac{m_{j}^{2}(v)}{v^{2}} T^{2} .
$$

Thus, we obtain the thermal mass from the two Higgs fields and Goldstone fields, respectively, as

$$
\begin{aligned}
\pi_{\text {scalar }}^{\text {Higgl } 1}(0) & =\frac{1}{4}\left(\lambda_{R 1}+\lambda_{R 2}\right) T^{2}, \\
\pi_{\text {scalar }}^{\text {Gold }}(0) & =\frac{1}{12}\left(\lambda_{R 1}+\lambda_{R 2}\right) T^{2} .
\end{aligned}
$$

Similarly, the scalar thermal mass contributions from the gauge fields are obtained in the following:

$$
\begin{aligned}
& \pi_{\text {scalar }}^{\text {lepto }}(0)=\frac{1}{48} g_{4}^{2} T^{2}, \quad \pi_{\text {scalar }}^{W_{R}^{ \pm}}(0)=\frac{1}{48} g_{R}^{2} T^{2} \\
& \pi_{\text {scalar }}^{Z^{\prime}}(0)=\frac{1}{96}\left(2 g_{R}^{2}+3 g_{4}^{2}\right) T^{2} .
\end{aligned}
$$

To obtain the total thermal mass contributions to the Higgs field, we need to include all the above thermal masses, i.e., Eqs. (31) and (32), and we have 


$$
\begin{aligned}
\sum_{j} \pi_{\text {scalar }}^{j}(0)= & \pi_{\text {scalar }}^{\text {Higgs1 }}(0)+6 \pi_{\text {scalar }}^{\text {Higgs2 }}(0)+9 \pi_{\text {scalar }}^{\text {Gold }}(0) \\
& +18 \pi_{\text {scalar }}^{\text {lepto }}(0)+6 \pi_{\text {scalar }}^{W_{R}^{ \pm}}(0)+3 \pi_{\text {scalar }}^{Z^{\prime}}(0) .
\end{aligned}
$$

Note that for each scalar field d.o.f. (either the Higgs or Goldstone bosons), it receives the same ring diagram contributions $\sum_{j} \pi_{i}^{j}$. Thus, by using Eq. (29) and Eq. (33), we obtain the total ring contributions to the scalar fields in the Pati-Salam model:

$$
V_{\text {ring }}^{\text {scalar,tot }}=V_{\text {ring }}^{\mathrm{Higgs} 1}+6 V_{\text {ring }}^{\mathrm{Higgs} 2}+9 V_{\text {ring }}^{\mathrm{Gold}} .
$$

Now we consider the case where the gauge fields are in the central ring of the Daisy diagram. We have the following general formulas to calculate the gauge, scalar, and fermion fields contributions to the gauge thermal masses for both Abelian and non-Abelian cases (see, e.g., [35]). For the Abelian case, we have

$$
U(1): \pi_{\text {gauge }}^{L, S}=\frac{g^{\prime 2} T^{2}}{3} \sum_{S} Y_{S}^{2}, \quad \pi_{\text {gauge }}^{L, F}=\frac{g^{\prime 2} T^{2}}{6} \sum_{F} Y_{F}^{2},
$$

where $L$ denotes the longitudinal thermal mass since it can be shown that the transverse thermal mass is suppressed and $Y_{S}, Y_{F}$ corresponds, respectively, to the hypercharge of relevant scalar and fermion fields. For the non-Abelian case, we have

$$
\begin{aligned}
S U(N): \pi_{\text {gauge }}^{L, S} & =\frac{g^{2} T^{2}}{3} \sum_{S} t_{2}\left(R_{S}\right), \\
\pi_{\text {gauge }}^{L, F} & =\frac{g^{2} T^{2}}{6} \sum_{F} t_{2}\left(R_{F}\right), \\
\pi_{\text {gauge }}^{L, V} & =\frac{N}{3} g^{2} T^{2}
\end{aligned}
$$

where $t_{2}\left(R_{S}\right), t_{2}\left(R_{F}\right)$ corresponds, respectively, to the Dynkin indices of the scalar and fermion representations, $\operatorname{Tr}\left[T_{R}^{a} T_{R}^{b}\right]=t_{2}(R) \delta^{a b}$. We obtain the total thermal mass contributions to the leptoquark, $W_{R}^{ \pm}$, and $Z^{\prime}$ are respectively

$\pi_{\text {lepto }}^{L, \text { Tot }}=\frac{5}{3} g_{4}^{2} T^{2}, \quad \pi_{W_{R}^{ \pm}}^{L, \text { Tot }}=\frac{4}{3} g_{R}^{2} T^{2}, \quad \pi_{Z^{\prime}}^{L, \text { Tot }}=\frac{4}{3} g_{4}^{2} T^{2}$.

When computing ring contributions for gauge fields, we use the original basis instead of the mass eigenstates. Thus, both $m_{i}^{2}(\rho)$ and $\sum_{i} \pi_{i}^{j}(0)$ are rewritten as matrices $\mathbf{M}^{2}(\rho)$ and $\boldsymbol{\Pi}(\mathbf{0})$, respectively, rather than eigenvalues as in the above scalar case. Equation (30) can be correspondingly modified as
$V_{\text {ring }}^{\text {gauge,tot }}=-\frac{T}{12 \pi} \operatorname{Tr}\left(\left[\mathbf{M}^{2}(\rho)+\mathbf{\Pi}(\mathbf{0})\right]^{3 / 2}-\mathbf{M}^{3}(\rho)\right)$,

where we include all contributions to the gauge rings and take into account only the massive gauge bosons for the big rings. The $\boldsymbol{\Pi}(\mathbf{0})$ is a diagonal 10-by-10 matrix with the entries of $(i, i)$ being $5 g_{4}^{2} T^{2} / 3$ and entries of $(j, j)$ being $4 g_{R}^{2} T^{2} / 3$ for $i=(1, \ldots, 7)$ and $j=(8,9,10)$.

In contrast, $\mathbf{M}^{2}(\rho)$ is a nearly diagonal symmetric 10-by10 matrix with the first six diagonal elements being $g_{4}^{2} \rho^{2} / 4$, the seventh being $3 g_{4}^{2} \rho^{2} / 8$, and the last three diagonal being $g_{R}^{2} \rho^{2} / 4$, plus two off-diagonal elements: $\left[\mathbf{M}^{2}(\rho)\right]_{7,10}=$ $\left[\mathbf{M}^{2}(\rho)\right]_{10,7}=\sqrt{3 / 32} g_{R} g_{4} \rho^{2}$.

\section{E. Complete finite-temperature potential}

Now we are ready to write out the total finite-temperature effective potential of the Pati-Salam model. It can be written as

$$
V_{\text {tree }}+V_{\text {lloop }}+V_{T}^{\text {tot }}+V_{\text {ring }}^{\text {scalar,tot }}+V_{\text {ring }}^{\text {gauge,tot }}
$$

\section{FIRST ORDER PHASE TRANSITION AND GRAVITATIONAL WAVE}

In this section, we will discuss the order of the possible early time Pati-Salam phase transition and the impact on possible gravitational wave signals.

\section{A. Strong first order phase transition}

Here we focus on showing that a strong first order phase transition can occur at around the Pati-Salam symmetry breaking scale with sample coupling solutions shown in Table II. We did not include all the couplings in the table since the remaining couplings are irrelevant in the analysis of our effective potential. We further note that the sample solutions in Table II are the ones leading to an asymptotically safe extension of the Pati-Salam model. However, we will show that the occurrence of a first order phase transition is not limited to this set of specific values of the couplings.

The finite-temperature effective potential equation (39) is shown in Fig. 1. Here we have set the renormalization scale $\mu$ at $5000 \mathrm{TeV}$, which is reasonable as the lower bound

TABLE II. This table summarizes the sample coupling solutions at the Pati-Salam symmetry breaking scale. We did not include $\lambda_{1}, \lambda_{2}, \lambda_{3}, \lambda_{4}, \lambda_{R \Phi_{1}}, \lambda_{R \Phi_{2}}, \lambda_{R \Phi_{3}}, y, y_{c}$ since they are irrelevant in studying the finite-temperature effective potential. Note that this set of solutions is obtained from a safe UV fixed point.

\begin{tabular}{lcccccc}
\hline \hline$\alpha_{L}$ & $\alpha_{R}$ & $\alpha_{4}$ & $\lambda_{R 1}$ & $\lambda_{R 2}$ & $y_{F}$ & $y_{\nu}$ \\
\hline 0.0038 & 0.0015 & 0.0109 & 0.291 & -0.291 & 0.004 & 0.645 \\
\hline \hline
\end{tabular}




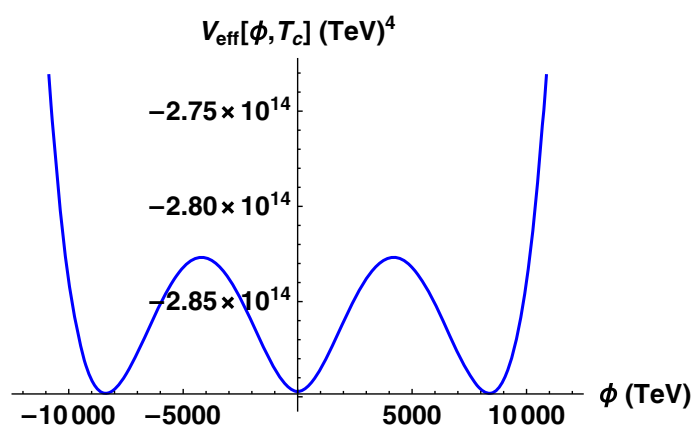

FIG. 1. We plot the finite-temperature effective potential by using the set of the couplings in Table II. The renormalization scale $\mu$ is set at $5000 \mathrm{TeV}$ while the temperature is chosen at $T=T_{c}=2680 \mathrm{TeV}$ which is the critical temperature.

on the Pati-Salam physics scale is at $2000 \mathrm{TeV}$ or so, derived from the upper limit $\operatorname{Br}\left(K_{L} \rightarrow \mu^{ \pm} e^{\mp}\right)<4.7 \times$ $10^{-12}$ [36]. We have also chosen the temperature $T$ to match the critical temperature, i.e., $T=T_{c}=2680 \mathrm{TeV}$ at which the potential has degenerate minima.

A positive nontrivial (away from the origin) minimum occurs for $\phi_{R} \sim 8400 \mathrm{TeV}$ and it is denoted as $\phi_{R c}$ and thus $\phi_{R c} / T_{c} \sim 3.13>1$. This shows that the associated phase transition is a strong first order one.

\section{B. Connection between first order phase transition and Coleman-Weinberg symmetry breaking}

We noticed that a strong first order phase transition occurs when spontaneous symmetry breaking happens via the Coleman-Weinberg mechanism. This is in line with the results and expectations of [33] (see also relevant discussions in, e.g., [37]). Of course, in other models first order phase transitions can still occur when symmetry breaking is generated via a hard negative mass square in the potential [38].

Around the finite-temperature transition, the ColemanWeinberg values of the couplings reported in Table II are such that $\lambda_{R 1} \simeq-\lambda_{R 2}$, canceling each other. From the RG flow point of view, in Fig. 2, Coleman-Weinberg symmetry breaking occurs when the RG flows of $\lambda_{R 1}(\mu)+\lambda_{R 2}(\mu)$ run

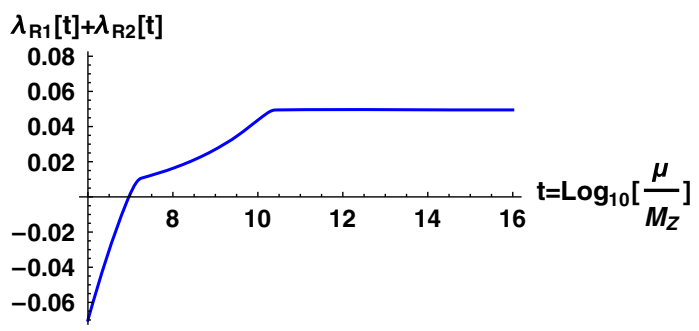

FIG. 2. We plot the RG running of $\lambda_{R 1}(t)+\lambda_{R 2}(t)$ from UV to IR. The transition point [the scale $\lambda_{R 1}(t)+\lambda_{R 2}(t)=0$ ] defines the Coleman-Weinberg symmetry breaking scale of the PatiSalam model.

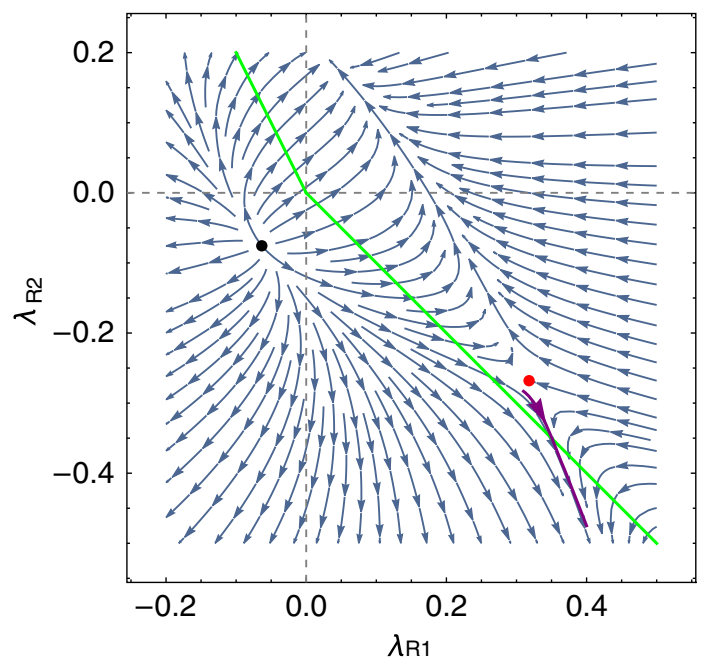

FIG. 3. We show the stream plot of $\lambda_{R 1}, \lambda_{R 2}$ where the flow direction is defined from UV to IR. The red and black plots are both the fixed point. The two green lines are the symmetry breaking lines which are defined as $\lambda_{R 1}+\lambda_{R 2}=0$ for $\lambda_{R 2}<0$ and $\lambda_{R 2} / 2+\lambda_{R 1}=0$ for $\lambda_{R 2}>0$. The purple line is the particular $\mathrm{RG}$ flow corresponding to the sample solution in Table II.

from positive to negative flowing from the UV to the IR. The transition point [the scale $\lambda_{R 1}(\mu)+\lambda_{R 2}(\mu)=0$ ] defines the dynamical symmetry breaking scale of the Pati-Salam model which is below $10000 \mathrm{TeV}$.

To gain insight it is interesting to show the symmetry breaking phenomenon via the stream plot provided in Fig. 3. The green line consisting of two symmetry breaking lines $\left(\lambda_{R 1}+\lambda_{R 2}=0\right.$ for $\lambda_{R 2}<0$ and $\lambda_{R 2} / 2+\lambda_{R 1}=0$ for $\left.\lambda_{R 2}>0\right)$ divides the plot into two phases. The right-hand side of the green line corresponding to the vacuum stable phase while the left side is related to the symmetry breaking phase. In our convention the arrows point towards the infrared. The two dots correspond, respectively, to a saddle point (the red one) and to an UV fixed point in both couplings. The bare couplings are meant to be fixed at some high energy scale on the right-hand side of the plot. A glance at the plot shows that the only consistent way to radiatively cross the green line is by initiating the flow in the bottom right corner of the plot. One might be tempted to cross it from left to right by starting near the black dot. However, this scenario would lead to an unstable potential at high energies and therefore is discarded.

Focusing on the bottom right corner, there is a special asymptotically safe trajectory emanating from the red dot. On that trajectory the theory will avoid a Landau pole and can be considered fundamental (up to gravity) in the deep ultraviolet. Another point is that the trajectory leads to a predictive infrared physics. We are also pleased to see that there is a wider region of UV bare coupling values that lead to a Coleman-Weinberg phenomenon beyond the asymptotically safe limit. 


\section{Bubble nucleation}

The time is ripe to discuss bubble nucleation within our model. We will provide a brief review of the method and apply it to our case.

The general picture is that as the Universe cools down, a second minimum, away from the origin, develops below a critical temperature. This triggers the tunneling from the false vacuum, at the origin, to the stable vacuum below the critical temperature. Assuming the transition to be first order, the tunneling rate per unit volume $\Gamma(T)$ from the metastable (false) vacuum to the stable one is suppressed by the three-dimensional Euclidean action $S_{3}(T)$ and we have [39-41] (for more recent work see, e.g., [42])

$$
\Gamma(T)=\left(\frac{S_{3}(T)}{2 \pi T}\right)^{3 / 2} T^{4} e^{-S_{3}(T) / T} .
$$

The Euclidean action has the form:

$$
S_{3}(\rho, T)=4 \pi \int_{0}^{\infty} d r r^{2}\left[\frac{1}{2}\left(\frac{d \rho}{d r}\right)^{2}+V(\rho, T)-V(0, T)\right]
$$

where we use the difference of the potential $F(\rho, T) \equiv$ $V(\rho, T)-V(0, T)$ to adjust the "datum point" of the potential at zero. The bubble configuration (instanton solution) is given by solving the following equation of motion of the action in Eq. (41):

$$
\frac{d^{2} \rho}{d r^{2}}+\frac{2}{r} \frac{d \rho}{d r}-\frac{\partial F}{\partial \rho}(\rho, T)=0
$$

with the associated boundary conditions:

$$
\frac{d \rho}{d r}(0, T)=0, \quad \lim _{r \rightarrow \infty} \rho(r, T)=0 .
$$

To find the solutions we use the so-called overshooting and undershooting method. We also used the numerical package, CosmoTransitions [43], to cross-check ${ }^{8}$ our results. For $T=2200 \mathrm{TeV}$ the bubble profile is shown in Fig. 4 . We can insert the bubble profile $\rho(r, T)$ into the Euclidean action equation (41) and thus $S_{3}$ will be dependent on $T$ only.

\footnotetext{
${ }^{8}$ It should be noted that although our scalar potential $V\left(\phi_{R}\right)$ for Pati-Salam symmetry breaking consists of multifields, the vacuum expectation values can be rotated to one single field component under the rotation of fundamental $S U(4)_{c} \times$ $S U(2)_{R}$. Thus, the scalar potential can be treated as a onedimensional potential which is suitable for CosmoTransitions package. However, for multiple scalar fields under the different symmetry group, the symmetry rotation will not help and we believe the more recent package known as "Anybubble" [44] should be very helpful.
}

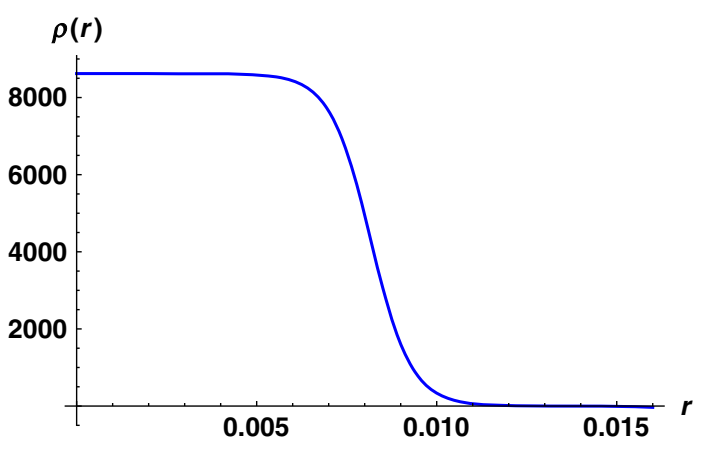

FIG. 4. We plot the bubble profile $\rho(r)$, where $T$ is chosen at $T=2200 \mathrm{TeV}$ which is slightly lower than the critical temperature at $T_{c}=2680 \mathrm{TeV}$.

The next step is to obtain the nucleation temperature which is defined as the temperature at which the rate of bubble nucleation per Hubble volume and time is approximately one. This means

$$
\Gamma(T) \sim H^{4},
$$

where $H$ is the Hubble constant. By using Eq. (40), we obtain

$$
T \ln \frac{T}{m_{\mathrm{pl}}} \simeq-\frac{S_{3}(T)}{4}
$$

where $m_{\mathrm{pl}}$ is the Planck mass. By solving Eq. (45) numerically, we find the nucleation temperature $T_{n}$ is around $1260 \mathrm{TeV}$. The inverse duration of the phase transition $\beta$ relative to the Hubble rate $H_{*}$ at the nucleation temperature $T_{n}$ is given by:

$$
\frac{\beta}{H_{*}}=\left.\left[T \frac{d}{d T}\left(\frac{S_{3}(T)}{T}\right)\right]\right|_{T=T_{n}} .
$$

We numerically obtain $\beta / H_{*} \simeq 183$.

Next, we will calculate another important parameter $\alpha$ which is the ratio of the latent heat released by the phase transition normalized against the radiation density:

$$
\begin{aligned}
\alpha & =\frac{\epsilon}{\rho_{\mathrm{rad}}}=\frac{1}{\frac{\pi^{2}}{30} g_{*} T_{n}^{4}}\left(-\Delta V+T_{n} \Delta s\right) \\
\Delta V & =V\left(v_{T_{n}}, T_{n}\right)-V\left(0, T_{n}\right) \\
\Delta s & =\frac{\partial V}{\partial T}\left(v_{T_{n}}, T_{n}\right)-\frac{\partial V}{\partial T}\left(0, T_{n}\right),
\end{aligned}
$$

where $v_{T_{n}}$ is the vacuum expectation value of the finitetemperature effective potential at the nucleation temperature, and $g_{*}(=150)$ is the relativistic d.o.f. in the universe. We find $\alpha_{T_{n}} \equiv \alpha\left(T=T_{n}\right)=0.217$. 


\section{Gravitational waves}

We now have all the instruments to address the generation and potential observation of gravitational waves stemming from the Pati-Salam early times phase transition.

For the reader's benefit, we provide a brief review of the ingredients needed to discuss the acoustic gravitational waves signals by following Ref. [45]. The discussion about collision dynamics of scalar field shells and turbulence can be found in [45] and their effects can be safely neglected in light of being subleading.

The power spectrum of the acoustic gravitational wave is given by ${ }^{9}$

$h^{2} \Omega_{s w}(f)=8.5 * 10^{-6}\left(\frac{100}{g_{*}}\right)^{\frac{1}{3}} \Gamma_{\mathrm{AI}}^{2} \bar{U}_{f}^{4}\left(\frac{H_{*}}{\beta}\right) v_{w} S_{s w}(f)$,

where the adiabatic index $\Gamma_{\mathrm{AI}}=\bar{\omega} / \bar{\epsilon} \simeq 4 / 3 . \bar{\omega}$ and $\bar{\epsilon}$ denote, respectively, the volume-averaged enthalpy and energy density respectively. $\bar{U}_{f}$ is a measure of the rootmean-square fluid velocity and is given by

$$
\bar{U}_{f}^{2} \simeq \frac{3}{4} \kappa_{f} \alpha_{T_{n}}
$$

where $\kappa_{f}$ is the efficiency parameter and it is well approximated by

$$
\kappa_{f} \sim \frac{\alpha}{0.73+0.083 \sqrt{\alpha}+\alpha}
$$

when $v_{\omega}($ wall speed $) \rightarrow 1$. The spectral shape $S_{s w}(f)$ is given by

$$
S_{s w}(f)=\left(\frac{f}{f_{s w}}\right)^{3}\left(\frac{7}{4+3\left(f / f_{s w}\right)^{2}}\right)^{\frac{7}{2}}
$$

with peak frequency $f_{s w}$ approximated by

\footnotetext{
${ }^{9}$ Note that in $[46,47]$, it was claimed that there could be an impact of the suppression due to the shorter duration of the sound waves. Following [46], the suppression is written as$$
H \tau_{\mathrm{sh}} \sim \frac{(8 \pi)^{\frac{1}{3}} \operatorname{Max}\left(v_{w}, c_{s}\right)}{\bar{U}_{f}}\left(\frac{\beta}{H}\right)^{-1} .
$$

For $\beta / H \sim 180, \alpha \sim 0.2$ and $v_{w}=1$, Eq. (48) roughly leads to a factor of 10 suppression which is relatively small compared to the cases with ultra supercooling. The reason we do not have a huge suppression is our $\beta / H$ as well as $\alpha$ are not too big. It will cause a huge suppression if either of the two becomes much bigger. Nevertheless, a factor of 10 suppression will not change our main conclusions, i.e., our predictions of the GW signals can be tested by the near future GW experiments.
}

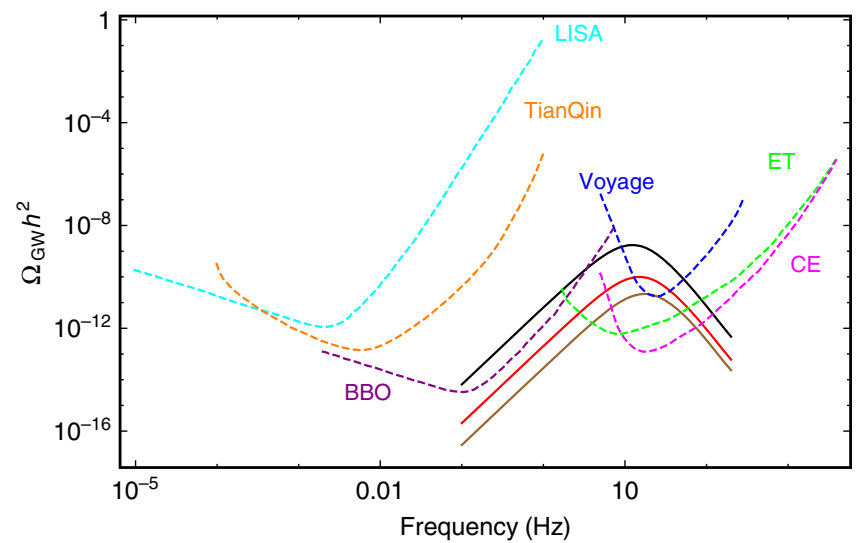

FIG. 5. In this diagram, we show the bounds of the relevant future gravitational wave detections in the plot of dimensionless energy density in gravitational waves against frequency. The bounds of LIGO Voyager, LISA, TianQing, BBO, Einstein Telescope (ET), Cosmic Explorer (CE) are shown, respectively, in blue, cyan, orange, purple, green, and magenta. The acoustic gravitational wave signals predicted in our Pati-Salam models are shown in black, red, and brown. The red one is predicted in the asymptotically safe scenario while the black and brown ones are with the Yukawa coupling $\left(y_{F}, y_{\nu}\right)$ values deviated from the safe scenario without modifying the IR SM physics.

$f_{s w}=8.9 \mu \mathrm{Hz} \frac{1}{v_{\omega}}\left(\frac{\beta}{H_{*}}\right)\left(\frac{z_{p}}{10}\right)\left(\frac{T_{n}}{100 \mathrm{GeV}}\right)\left(\frac{g_{*}}{100}\right)^{\frac{1}{6}}$

with $z_{p}$ a simulation-derived factor that is of order 10 , and following [48] we take it to be 6.9.

By substituting $\alpha_{T_{n}}$ and $\beta / H_{*}$ from Eq. (46) and Eq. (47) into the above power spectrum formula for the acoustic gravitational wave equation (49), we plot the curves of the energy density against frequency (solid lines and the sample solution in Table II is in red) in Fig. 5 where the coupling solutions in Table IV are used. We have also included the future bounds (dashed lines) coming from planned gravitational wave detection experiments such as LIGO Voyager [49,50], LISA [4], TianQing [51], BBO [52,53], ET [54,55], and CE [49]. They are shown, respectively, in blue, cyan, orange, purple, green, and magenta in Fig. 5. Interestingly, we find the predicted acoustic gravitational wave signal predicted to be within the detection region of the LIGO Voyager which is planned to be operational around 2027-2028. Note that a novel method for presenting and comparing the gravitational wave signals from different models has been proposed in [47].

\section{PATI-SALAM DRIVEN GRAVITY WAVES}

We are now in a position to analyze in more detail the parameter space of bare couplings leading to observable gravitational waves within the Pati-Salam grand unified framework. 
For convenience we start with the asymptotically safe Pati-Salam scenario that has helped us quickly identify the relevant parameter space for the occurrence of a strong first order phase transition.

\section{A. Asymptotically safe case}

In this section, we discuss an asymptotically safe embedding of the Pati-Salam framework by adding a large number of vectorlike fields into the theory. In this limit we will argue for the existence of an UV fixed point which solves the triviality problem while yielding a highly predictive theory at lower energies.

Without further ado we introduce $N_{F}$ pairs of vectorlike fermions charged under the fundamental representation of the Pati-Salam gauge group equation (1) with the following charge assignments:

$$
N_{F}:(4,1,2) \oplus(4,2,1) \text {. }
$$

For simplicity, we assume that these new vectorlike fermions appear at the Pati-Salam symmetry breaking scale.

Employing the large- $N_{F}$ beta functions reported in the Appendix, we can compute the RG flow connecting the UV fixed point (red dot in Fig. 3) and the SM in the infrared. For each $N_{F} \gg 1$ input, we obtain a set of UV fixed point solutions. Follow the RG flow starting from the determined UV fixed point to the electroweak scale, we can check whether it matches onto the SM.

At the PS symmetry breaking scale, we need to use matching conditions for both the gauge couplings and scalar quartic couplings. In particular, after PS symmetry breaking, the scalar bidoublet should match the conventional two Higgs doublet model (we implement the beta functions of the two Higgs doublet model provided in [56]). We have searched the full parameter space in the range of $N_{F} \in(10,200)$ and find that $N_{F}=13$ with the UV fixed point solutions shown in Table III agree best with the low energy data (both the Higgs mass and the top Yukawa coupling at the electroweak scale). We note that $y_{F}$ is asymptotically free for all viable solutions. We have therefore provided a UV safe completion of the SM. ${ }^{10}$

The sample solutions in Table II are already the asymptotically safe solutions corresponding to $N_{F}=13$. This set is particularly interesting because of the following:

(i) It corresponds to a possible UV safe fixed point rendering (up to gravity) our Pati-Salam model UV complete.

\footnotetext{
${ }^{10}$ We note that even if the fixed point is not entirely established, this analysis is still valid because the associated trajectories are valid for any energy scale sufficiently close to the would-be UV fixed point due to the nature of the precise results of the large- $N_{f}$ expansion away from the fixed point.
}

TABLE III. This table summarizes the UV fixed point solution for $N_{F}=13$ involving the bubble diagram contributions in the Yukawa and quartic RG beta functions. $y_{F}$ is asymptotically free and thus is zero at the fixed point.

\begin{tabular}{lcccccccccc}
\hline \hline$\lambda_{1}$ & $\lambda_{2}$ & $\lambda_{3}$ & $\lambda_{4}$ & $\lambda_{R \Phi_{1}}$ & $\lambda_{R \Phi_{2}}$ & $\lambda_{R 1}$ & $\lambda_{R 2}$ & $y, y_{c}$ & $y_{F}$ & $y_{\nu}$ \\
\hline 0.13 & 0.01 & 0.03 & 0.05 & 0.10 & 0.01 & 0.34 & -0.29 & 0.53 & 0 & 0.67 \\
\hline \hline
\end{tabular}

(ii) The Pati-Salam symmetry is dynamically broken through the Coleman-Weinberg mechanism below $10000 \mathrm{TeV}$ (see Fig. 2) without adding any mass terms. ${ }^{11}$

(iii) Below $2680 \mathrm{TeV}$, a strong first order phase transition occurs and at the nucleation temperature $T_{n}=$ $1260 \mathrm{TeV}$ gravitational wave signals can be generated. These are within the reach of the planned LIGO Voyager experiment detection region see Fig. 5 and the signal-to-noise-ratio for the safe scenario is 30) as well as the detection regions envisioned for the ET, CE, and Big Bang Observer (BBO).

We show the results as the red solid curve in both Fig. 5 and Fig. 6.

\section{B. Beyond the safe scenario}

Here, we will go beyond the safe scenario by exploring a more general parameter space able to generate testable gravitational wave signals.

We observe that the gauge couplings $g_{4}, g_{R}, g_{L}$ are fixed by the Standard Model once the Pati-Salam symmetry breaking scale is chosen. In addition, when varying the quartic couplings we must ensure the presence of the Standard Model Higgs with its $125 \mathrm{GeV}$ mass at the electroweak scale. We therefore vary only the Yukawa couplings $y_{F}, y_{\nu}$ and the two quartic couplings $\lambda_{R 1}, g_{L}$ to satisfy this constraint.

Scanning the Yukawa coupling parameter space, we discover that when increasing either $y_{F}$ or $y_{\nu}$ (see black row of Table IV), the dimensionless energy density of the gravitational wave signal increases accordingly and the peak frequency will shift slightly to the left. This is clear when comparing the black curve with the red (safe) curve in Fig. 5.

When scanning the quartic couplings parameter space, we find that the gravitational waves signal also depends on $\lambda_{R 1}+\lambda_{R 2}$. Varying $\lambda_{R 1}, \lambda_{R 2}$ with fixed $\lambda_{R 1}+\lambda_{R 2}$, the dimensionless energy density and the peak of the frequency of the gravitational wave signals are roughly fixed. When increasing $\lambda_{R 1}+\lambda_{R 2}$ (see the Brown and Grey rows of Table V) the dimensionless energy density of the gravitational wave signal decreases accordingly and the peak

\footnotetext{
${ }^{11}$ This result does not depend on the existence of the fixed point but it is a welcome prediction.
} 


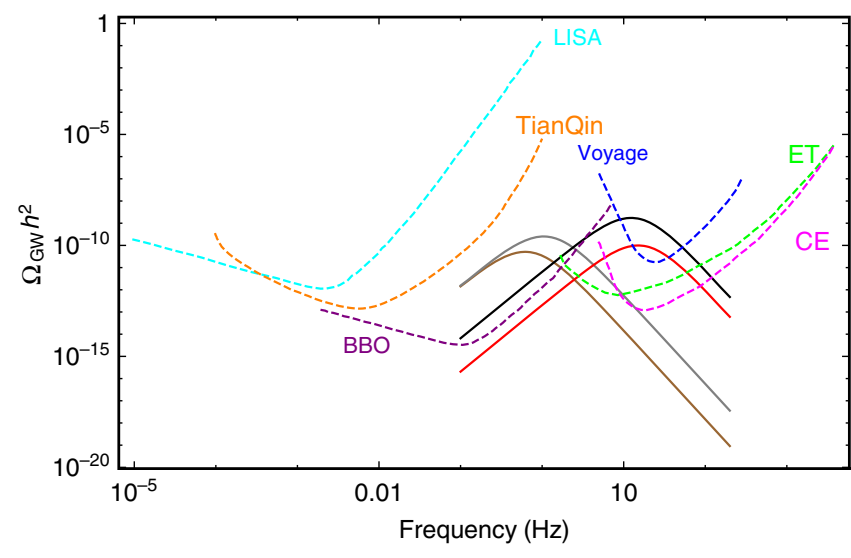

FIG. 6. In this diagram, we show the bounds of the relevant future gravitational wave detections in the plot of dimensionless energy density in gravitational waves against frequency. The bounds of LIGO Voyager, LISA, TianQing, BBO, ET, and CE are shown, respectively, in blue, cyan, orange, purple, green, and magenta. The acoustic gravitational wave signals predicted in our Pati-Salam models are shown in red, black, grey, and brown. The red one is predicted in the asymptotically safe scenario while the black, grey, and brown ones are with the Yukawa and Quartic coupling $\left(\lambda_{R 1}, \lambda_{R 2}\right)$ values deviated from the safe scenario without touching the IR SM physics.

frequency shifts significantly to the left with respect to the safe scenario. This can be seen from Fig. 6 .

Thus, different from the safe scenario where the peak frequency is roughly around $10 \mathrm{~Hz}$, going beyond the safe scenario allows for a peak of frequency ranging between 0.1 and $10 \mathrm{~Hz}$.

TABLE IV. This table summarizes the sample coupling solutions at the Pati-Salam symmetry breaking scale. Safe, Black, and Brown represent, respectively, the gravitational wave energy density curves with the Red, Black, and Brown colors in Fig. 5.

\begin{tabular}{lccccccc}
\hline \hline & $\alpha_{L}$ & $\alpha_{R}$ & $\alpha_{4}$ & $\lambda_{R 1}$ & $\lambda_{R 2}$ & $y_{F}$ & $y_{\nu}$ \\
\hline Safe & 0.0038 & 0.0015 & 0.0109 & 0.291 & -0.291 & 0.004 & 0.645 \\
Black & 0.0038 & 0.0015 & 0.0109 & 0.291 & -0.291 & 0.5119 & 0.645 \\
Brown & 0.0038 & 0.0015 & 0.0109 & 0.291 & -0.291 & 0.001 & 0.001 \\
\hline \hline
\end{tabular}

TABLE V. This table summarizes the sample coupling solutions at the Pati-Salam symmetry breaking scale. The Safe, Black, Brown, and Grey represent, respectively, the gravitational wave energy density curves with Red, Black, Brown, and Grey colors in Fig. 6.

\begin{tabular}{lccccccc}
\hline \hline & $\alpha_{L}$ & $\alpha_{R}$ & $\alpha_{4}$ & $\lambda_{R 1}$ & $\lambda_{R 2}$ & $y_{F}$ & $y_{\nu}$ \\
\hline Safe & 0.0038 & 0.0015 & 0.0109 & 0.291 & -0.291 & 0.004 & 0.645 \\
Black & 0.0038 & 0.0015 & 0.0109 & 0.291 & -0.291 & 0.5119 & 0.645 \\
Brown & 0.0038 & 0.0015 & 0.0109 & 0.291 & -0.001 & 0.5119 & 0.645 \\
Grey & 0.0038 & 0.0015 & 0.0109 & 0.291 & -0.093 & 0.5119 & 0.645 \\
\hline \hline
\end{tabular}

\section{CONCLUSIONS}

We investigated the gravitational wave signatures stemming from the Pati-Salam model by presenting a few benchmark points in the parameter space supporting a strong first order phase transition.

We started the analysis by employing a safe version of the Pati-Salam extension of the Standard Model and then quickly generalized to more generic situations. We find that a Coleman-Weinberg spontaneous breaking of the symmetry triggers a first order phase transition that can be observed via the next generation of gravitational wave detectors such as LIGO Voyager, the ET, and the CE.

Beyond the safe scenario we notice that the Yukawa couplings $y_{\nu}, y_{F}$ affect mostly the gravitational wave energy density while the combination of quartic couplings $\lambda_{R 1}+\lambda_{R 2}$ shifts its peak frequency.

Concluding, we discover that the peak frequency of the gravitational wave signals stemming from the Pati-Salam model ranges within $0.1-10 \mathrm{~Hz}$. Our results lead to the exciting news that the next generation of gravity wave detectors will be able to explore important extensions of the Standard Model appearing not at the electroweak scale but at much higher energy scales not accessible through present and future particle physics accelerators.

\section{ACKNOWLEDGMENTS}

The work is partially supported by the Danish National Research Foundation under Grant No. DNRF:90. W. C. H. was supported by the Independent Research Fund Denmark, Grant No. DFF 6108-00623. Z.-W. W. thanks Tom Steele, Robert Mann, and Steve Abel for helpful discussions and Huan Yang for recommending the relevant references. Z.-W. W. also thanks Tsutomu Yanagida's comments about the monopole issue.

\section{APPENDIX: LARGE- $N_{F}$ BETA FUNCTIONS}

The beauty of the large- $N_{F}$ beta function is noticing that a subset of the Feynman diagrams (denoted as bubble chain) can be summed up into a closed form at $1 / N_{F}$ order. Thus, all the higher order information up to $1 / N_{F}$ order is encoded in the summation functions denoted as $F_{1}(A)$, $H_{1}(A), H_{0}(A)$ below. It also serves to note that these summation functions possess the pole structures:

$$
F_{1}(A) \sim \log (1-2 A / 15), \quad H_{1}(A) \sim \log (1-A / 3),
$$

which guarantees the UV fixed point for the gauge beta functions and opens the possibility for the fixed point solutions for all the couplings.

To the leading $1 / N_{F}$ order, the higher order (ho) contributions to the general RG functions of the gauge couplings were computed in [20], while for the simple 
gauge groups they were computed in $[17,57]$ and for the Abelian in [16]. Here we summarize the results. The ho contributions to $d \alpha_{i} / d \log \mu$ (in the semi-simple case) are

$$
\begin{aligned}
\beta_{i}^{\text {ho }} & =\frac{2 A_{i} \alpha_{i}}{3}\left(\frac{d\left(R_{i}\right) H_{1_{i}}\left(A_{i}\right)}{N_{F_{i}} \prod_{k} d\left(R_{\psi}^{k}\right)}+\frac{\sum_{j} d\left(G_{j}\right) F_{1_{j}}\left(A_{j}\right)}{N_{F_{i}} \prod_{k} d\left(R_{\psi}^{k}\right)}\right), \\
\alpha_{i} & \equiv \frac{g_{i}^{2}}{(4 \pi)^{2}} \quad(i=L, R, C),
\end{aligned}
$$

with the functions $H_{1 i}$ and the t'Hooft couplings $A_{i}$

$$
\begin{aligned}
A_{i} & =4 \alpha_{i} T_{R} N_{F_{i}} \frac{\prod_{k} d\left(R_{\psi}^{k}\right)}{d\left(R_{\psi}^{i}\right)} \\
H_{1_{i}} & =\frac{-11}{4} \frac{C_{G}}{T_{R}}+\int_{0}^{A_{i} / 3} I_{1}(x) I_{2}(x) d x \\
F_{1_{j}} & =\int_{0}^{A_{j} / 3} I_{1}(x) d x
\end{aligned}
$$

where $I_{1}(x)$ and $I_{2}(x)$ are

$$
\begin{aligned}
I_{1}(x)= & \frac{(1+x)(2 x-1)^{2}(2 x-3)^{2} \sin (\pi x)^{3}}{(x-2) \pi^{3}} \\
& \times\left(\Gamma(x-1)^{2} \Gamma(-2 x)\right) \\
I_{2}(x)= & \frac{C_{R}}{T_{R}}+\frac{\left(20-43 x+32 x^{2}-14 x^{3}+4 x^{4}\right)}{4(2 x-1)(2 x-3)\left(1-x^{2}\right)} \frac{C_{G}}{T_{R}} .
\end{aligned}
$$

The Dynkin indices are $T_{R}=1 / 2\left(N_{c_{i}}\right)$ for the fundamental (adjoint) representation while $d\left(R_{\psi}^{k}\right)$ denotes the dimension of the fermion representation.

The RG functions of the (semisimple) gauge couplings are

$$
\begin{aligned}
\beta_{\alpha_{2 L}}^{\mathrm{tot}}= & \frac{d \alpha_{2 L}}{d \log \mu}=\beta_{\alpha_{2 L}}^{\text {loop }}+\beta_{\alpha_{2 L}}^{\text {ho }}=-6 \alpha_{2 L}^{2} \\
& +\frac{2 A_{2 L} \alpha_{2 L}}{3}\left(1+\frac{H_{1_{2 L}}\left(A_{2 L}\right)}{4 N_{F}}+\frac{15}{8} \frac{F_{1_{4}}\left(A_{4}\right)}{N_{F}}\right) \\
\beta_{\alpha_{2 R}}^{\mathrm{tot}}= & \frac{d \alpha_{2 R}}{d \log \mu}=\beta_{\alpha_{2 R}}^{\text {loop }}+\beta_{\alpha_{2 R}}^{\text {ho }}=-\frac{14}{3} \alpha_{2 R}^{2} \\
& +\frac{2 A_{2 R} \alpha_{2 R}}{3}\left(1+\frac{H_{1_{2 R}}\left(A_{2 R}\right)}{4 N_{F}}+\frac{15}{8} \frac{F_{1_{4}}\left(A_{4}\right)}{N_{F}}\right) \\
\beta_{\alpha_{4}}^{\text {tot }}= & \frac{d \alpha_{4}}{d \log \mu}=\beta_{\alpha_{4}}^{1 \text { loop }}+\beta_{\alpha_{4}}^{\text {ho }}=-18 \alpha_{4}^{2} \\
& +\frac{2 A_{4} \alpha_{4}}{3}\left(1+\frac{H_{1_{4}}\left(A_{4}\right)}{4 N_{F}}+\sum_{i=L / R} \frac{3}{16}\left(\frac{F_{1_{2 i}}\left(A_{2 i}\right)}{N_{F}}\right)\right) .
\end{aligned}
$$

The Yukawa beta function reads

$$
\begin{gathered}
\beta_{y}=c_{1} y^{3}+y \sum_{\alpha} c_{\alpha} g_{\alpha}^{2} I_{y}\left(A_{\alpha}\right), \quad \text { with } \\
I_{y}\left(A_{\alpha}\right)=H_{\phi}\left(0, \frac{2}{3} A_{\alpha}\right)\left(1+A_{\alpha} \frac{C_{2}\left(R_{\phi}^{\alpha}\right)}{6\left(C_{2}\left(R_{\chi}^{\alpha}\right)+C_{2}\left(R_{\xi}^{\alpha}\right)\right)}\right) \\
H_{\phi}(x)=H_{0}(x)=\frac{\left(1-\frac{x}{3}\right) \Gamma(4-x)}{3 \Gamma^{2}\left(2-\frac{x}{2}\right) \Gamma\left(3-\frac{x}{2}\right) \Gamma\left(1+\frac{x}{2}\right)}
\end{gathered}
$$

containing information about the resumed fermion bubbles and $c_{1}, c_{\alpha}$ are the standard one-loop coefficients for the Yukawa beta function while $C_{2}\left(R_{\phi}^{\alpha}\right), C_{2}\left(R_{\chi}^{\alpha}\right), C_{2}\left(R_{\xi}^{\alpha}\right)$ are the Casimir operators of the corresponding scalar and fermion fields. Thus, when $c_{1}, c_{\alpha}$ are known, the full Yukawa beta function follows. Similarly, for the quartic coupling we write

$$
\begin{aligned}
\beta_{\lambda}= & c_{1} \lambda^{2}+\lambda \sum_{\alpha} c_{\alpha} g_{\alpha}^{2} I_{\lambda g^{2}}\left(A_{\alpha}\right)+\sum_{\alpha} c_{\alpha}^{\prime} g_{\alpha}^{4} I_{g^{4}}\left(A_{\alpha}\right) \\
& +\sum_{\alpha<\beta} c_{\alpha \beta} g_{\alpha}^{2} g_{\beta}^{2} I_{g_{1}^{2} g_{2}^{2}}^{\mathrm{tot}}\left(A_{\alpha}, A_{\beta}\right),
\end{aligned}
$$

and with $c_{1}, c_{\alpha}, c_{\alpha}^{\prime}, c_{\alpha \beta}$ the known one-loop coefficients for the quartic beta function and the resumed fermion bubbles appear via

$$
\begin{aligned}
I_{\lambda g^{2}}\left(A_{\alpha}\right)= & H_{\phi}\left(0, \frac{2}{3} A_{\alpha}\right) \\
I_{g^{4}}\left(A_{\alpha}\right)= & H_{\lambda}\left(1, \frac{2}{3} A_{\alpha}\right)+A_{\alpha} \frac{d H_{\lambda}\left(1, \frac{2}{3} A_{\alpha}\right)}{d A_{\alpha}} \\
I_{g_{1}^{2} g_{2}^{2}}^{\text {tot }}\left(A_{\alpha}, A_{\beta}\right)= & \frac{1}{3}\left[I_{g_{1}^{2} g_{2}^{2}}\left(A_{\alpha}, 0\right)+I_{g_{1}^{2} g_{2}^{2}}\left(0, A_{\beta}\right)\right. \\
& \left.+I_{g_{1}^{2} g_{2}^{2}}\left(A_{\alpha}, A_{\beta}\right)\right] \\
I_{g_{1}^{2} g_{2}^{2}}\left(A_{\alpha}, A_{\beta}\right)= & \frac{1}{A_{\alpha}-A_{\beta}}\left[A_{\alpha} H_{\lambda}\left(1, \frac{2}{3} A_{\alpha}\right)\right. \\
& \left.-A_{\beta} H_{\lambda}\left(1, \frac{2}{3} A_{\beta}\right)\right], \\
H_{\lambda}(1, x)= & \left(1-\frac{x}{4}\right) H_{0}(x) \\
= & \frac{\left(1-\frac{x}{4}\right)\left(1-\frac{x}{3}\right) \Gamma(4-x)}{3 \Gamma^{2}\left(2-\frac{x}{2}\right) \Gamma\left(3-\frac{x}{2}\right) \Gamma\left(1+\frac{x}{2}\right)} .
\end{aligned}
$$

We therefore have the quartic beta function including the bubble diagram contributions when $c_{1}, c_{\alpha}, c_{\alpha}^{\prime}, c_{\alpha \beta}$ are known. 
[1] C. Grojean and G. Servant, Phys. Rev. D 75, 043507 (2007).

[2] R. Apreda, M. Maggiore, A. Nicolis, and A. Riotto, Nucl. Phys. B631, 342 (2002).

[3] M. Jarvinen, C. Kouvaris, and F. Sannino, Phys. Rev. D 81, 064027 (2010).

[4] C. Caprini et al., J. Cosmol. Astropart. Phys. 04 (2016) 001.

[5] C. Caprini et al., J. Cosmol. Astropart. Phys. 03 (2020) 024.

[6] J. C. Pati and A. Salam, Phys. Rev. D 10, 275 (1974); 11, 703(E) (1975).

[7] K. S. Babu, X. G. He, and S. Pakvasa, Phys. Rev. D 33, 763 (1986).

[8] D. Croon, T. E. Gonzalo, and G. White, J. High Energy Phys. 02 (2019) 083.

[9] A. Greljo, T. Opferkuch, and B. A. Stefanek, Phys. Rev. Lett. 124, 171802 (2020).

[10] P. Fileviez Perez and S. Ohmer, Phys. Lett. B 768, 86 (2017).

[11] J. C. Pati, Int. J. Mod. Phys. A 32, 1741013 (2017).

[12] A. H. Guth and E. J. Weinberg, Nucl. Phys. B212, 321 (1983).

[13] E. W. Kolb and M. S. Turner, The Early Universe (AddisonWesley, Redwood City, 1990).

[14] R. R. Volkas, Phys. Rev. D 53, 2681 (1996).

[15] D. F. Litim and F. Sannino, J. High Energy Phys. 12 (2014) 178 .

[16] A. Palanques-Mestre and P. Pascual, Commun. Math. Phys. 95, 277 (1984).

[17] J. A. Gracey, Phys. Lett. B 373, 178 (1996).

[18] R. Mann, J. Meffe, F. Sannino, T. Steele, Z. W. Wang, and C. Zhang, Phys. Rev. Lett. 119, 261802 (2017).

[19] G. M. Pelaggi, A. D. Plascencia, A. Salvio, F. Sannino, J. Smirnov, and A. Strumia, Phys. Rev. D 97, 095013 (2018).

[20] O. Antipin, N. A. Dondi, F. Sannino, A. E. Thomsen, and Z. W. Wang, Phys. Rev. D 98, 016003 (2018).

[21] O. Antipin and F. Sannino, Phys. Rev. D 97, 116007 (2018).

[22] E. Molinaro, F. Sannino, and Z. W. Wang, Phys. Rev. D 98, 115007 (2018).

[23] Z. W. Wang, A. Al Balushi, R. Mann, and H. M. Jiang, Phys. Rev. D 99, 115017 (2019).

[24] F. Sannino, J. Smirnov, and Z. W. Wang, Phys. Rev. D 100, 075009 (2019).

[25] R. N. Mohapatra and G. Senjanovic, Phys. Rev. Lett. 44, 912 (1980).

[26] P. Minkowski, Phys. Lett. 67B, 421 (1977).

[27] T. Yanagida, Conf. Proc. C7902131, 95 (1979).

[28] M. Gell-Mann, P. Ramond, and R. Slansky, Conf. Proc. C790927, 315 (1979), https://arxiv.org/abs/1306.4669.

[29] T. P. Cheng, E. Eichten, and L. F. Li, Phys. Rev. D 9, 2259 (1974).
[30] E. Gildener, Phys. Rev. D 13, 1025 (1976).

[31] F. F. Hansen, T. Janowski, K. Langble, R. B. Mann, F. Sannino, T. G. Steele, and Z. W. Wang, Phys. Rev. D 97, 065014 (2018).

[32] M. E. Carrington, Phys. Rev. D 45, 2933 (1992).

[33] F. Sannino and J. Virkajrvi, Phys. Rev. D 92, 045015 (2015).

[34] T. Li and Y. Zhou, J. High Energy Phys. 07 (2014) 006.

[35] J. M. Cline, M. Jarvinen, and F. Sannino, Phys. Rev. D 78, 075027 (2008).

[36] D. Ambrose et al. (BNL Collaboration), Phys. Rev. Lett. 81, 5734 (1998).

[37] F. A. Chishtie, Z. R. Huang, M. Reimer, T. G. Steele, and Z. W. Wang, Phys. Rev. D 102, 076021 (2020).

[38] J. M. Cline and P. A. Lemieux, Phys. Rev. D 55, 3873 (1997).

[39] S. R. Coleman, Phys. Rev. D 15, 2929 (1977).

[40] C. G. Callan, Jr. and S. R. Coleman, Phys. Rev. D 16, 1762 (1977).

[41] A. D. Linde, Nucl. Phys. B216, 421 (1983).

[42] A. Kobakhidze, C. Lagger, A. Manning, and J. Yue, Eur. Phys. J. C 77, 570 (2017).

[43] C. L. Wainwright, Comput. Phys. Commun. 183, 2006 (2012).

[44] A. Masoumi, K. D. Olum, and J. M. Wachter, J. Cosmol. Astropart. Phys. 10 (2017) 022.

[45] D. J. Weir, Phil. Trans. R. Soc. A 376, 20170126 (2018).

[46] J. Ellis, M. Lewicki, and J. M. No, J. Cosmol. Astropart. Phys. 07 (2020) 050.

[47] T. Alanne, T. Hugle, M. Platscher, and K. Schmitz, J. High Energy Phys. 03 (2020) 004.

[48] M. Hindmarsh, S. J. Huber, K. Rummukainen, and D. J. Weir, Phys. Rev. D 96, 103520 (2017).

[49] B. P. Abbott et al. (LIGO Scientific Collaboration), Classical Quantum Gravity 34, 044001 (2017).

[50] K. Yagi and H. Yang, Phys. Rev. D 97, 104018 (2018).

[51] J. Luo et al. (TianQin Collaboration), Classical Quantum Gravity 33, 035010 (2016).

[52] K. Yagi and N. Seto, Phys. Rev. D 83, 044011 (2011); 95, 109901(E) (2017).

[53] E. Thrane and J. D. Romano, Phys. Rev. D 88, 124032 (2013).

[54] M. Punturo et al., Classical Quantum Gravity 27, 194002 (2010).

[55] S. Hild et al., Classical Quantum Gravity 28, 094013 (2011).

[56] G. C. Branco, P. M. Ferreira, L. Lavoura, M. N. Rebelo, M. Sher, and J. P. Silva, Phys. Rep. 516, 1 (2012).

[57] B. Holdom, Phys. Lett. B 694, 74 (2010). 